\title{
Feststoffhaushalt und Sedimenttransport - zentrale Zukunftsthemen in der österreichischen und internationalen Wasserwirtschaft
}

An den Fließgewässern Österreichs aber auch international und global geht die Schere zwischen Überschuss an Feststoffen (z. B. in Stauhaltungen) und Defizit (z. B. in freien Fließstrecken der Flüsse) mehr und mehr auf.

Gemäß einer UN-Studie liegt der Zeitpunkt, zu dem $80 \%$ des Nutzvolumens der Speicher für die Wasserkraftnutzung verlandet sind, zwischen 2035 (z. B. Asien) und 2080 (z. B. Europa, Russland, Südamerika). Andererseits tiefen sich die Flüsse in freien Fließstrecken in Österreich mit einer Rate von 0,5 bis $7 \mathrm{~cm} / J a h r$ ein, im Extremfall kann nach Erosion der Kiesschicht ein Sohldurchschlag ins Tertiär/Feinmaterial erfolgen, mit allen technischen, ökologischen und ökonomischen Konsequenzen. Die Donau zeigt auch in der rumänisch-bulgarischen Grenzstrecke sowie im Delta Sohleintiefungen und das Sedimentdefizit führt am Schwarzen Meer zu einer Küstenerosion von bis zu 17 m/ Jahr. Auf globaler Ebene weisen viele große Flüsse ebenfalls eine Abnahme der Feststofffracht auf (z. B. Gelber Fluss, Yangtze, Mississippi, Mekong).

Diese Ausgabe der ÖWAW umfasst 6 Beiträge, welche die Sedimenttransportmessung, die Sedimenttransportmodellierung sowie ein nationales, bilaterales und internationales Projekt zum Thema Feststoffhaushalt und Sedimenttransport vorstellen.

Der erste Artikel „Geschiebemessung in Österreich“ (Kreisler et al.) beschreibt die Grundlagen, Zielsetzungen, Messmethoden und Messstellen zur Erfassung des Geschiebetransportes in Österreich. Dabei werden direkte und indirekte Messmethoden mit deren Einsatzmöglichkeiten und -grenzen vorgestellt sowie Beispiele von Messergebnissen angeführt, die die raum-zeitliche Variabilität des Geschiebetransportes unterstreichen und die Notwendigkeit von integrativen, mehrere Methoden umfassenden Geschiebemessstellen betonen.

Lalk et al. beschäftigen sich im Beitrag „Monitoring, Analyse und Interpretation des Schwebstofftransportes an österreichischen Flüssen" mit dem Transport von Schwebstoffen in Fließgewässern. In den letzten Jahren wurde in Österreich eine neue Methode zur kontinuierlichen Erfassung des Schwebstofftransportes entwickelt und umgesetzt. Die erfassten Schwebstoffdaten werden überprüft und im Hydrographischen Jahrbuch jährlich publiziert. Der Beitrag analysiert die Schwebstoffkonzentrationen, Transportraten, Frachten und Spenden von 28 Messstellen für den Zeitraum 2009-2011 und vergleicht die Ergebnisse in Hinblick auf die Unterschiede in den Einzugsgebieten.

Im Artikel „Auswirkungen von Baggerungen und Verklappungen hochwasserbedingter Feinsedimentablagerungen am Fallbeispiel Winterhafen Linz/Donau“ untersuchen Haimann et al. anhand von Naturmessungen und numerischen Simulationen, welche Auswirkungen Baggerungen von hochwasserbedingten Feinsedimentablagerungen und deren anschließende Verklappung in den Hauptstrom der Donau haben. Die mehrdimensionale Hydrodynamik- und Sedimenttransportmodellierung ermöglicht die Simulation von unterschiedlichen Szenarien, die auf Basis von Untersuchungen des Makrozoobenthos und der Fische beurteilt werden. Die Naturmessungen und Modellierung erlauben die Ableitung von Empfehlungen, damit Baggerungen und Verklappungen von Feinsedimenten an der Donau zukünftig optimiert durchgeführt werden können.

Das österreichische Projekt „SED_AT - Feststoffhaushalt, Sedimenttransport und Flussmorphologie im Rahmen des Nationalen Gewässerbewirtschaftungsplans“ wird von Habersack et al. im vierten Beitrag vorgestellt. Da mehr als $50 \%$ der im nationalen Gewässerbewirtschaftungsplan 
untersuchten Gewässer ein Risiko der Zielverfehlung des guten ökologischen Zustandes bzw. des guten ökologischen Potenzials bis 2015 aufweisen, das durch hydromorphologische Belastungen zustande kommt, wurden im Rahmen des Projektes SED_AT die bestehenden Probleme in diesen Bereichen mithilfe von Stakeholderbefragungen, durch Literaturrecherche und ExpertInnenabschätzung erfasst und daraus der zukünftige Handlungsbedarf (Forschungsbedarf, Managementbedarf und rechtliche Belange) abgeleitet. Es konnte gezeigt werden, dass in jedem gewässerrelevanten Sektor (Wildbach- und Lawinenverbauung, Flussbau, Ökologie, Energiewirtschaft, Wasserstraßen und Landwirtschaft) Veränderungen im Feststoffhaushalt, Sedimenttransport und der Flussmorphologie Probleme verursachen ....und dass Handlungsbedarf in Richtung verbessertes Sedimentmanagement gegeben ist, wie die Erstellung von Einzugsgebietsbezogenen Feststoffmanagementkonzepten oder die Entwicklung von Maßnahmen zur Optimierung von Bauwerken in Richtung Sedimentkontinuum.

Dass das Thema Sedimenttransport nicht nur eine österreichspezifische Thematik darstellt, zeigt der Artikel „Gemeinsame österreichisch-ungarische Sedimentforschung im Rahmen des EFREProjektes SEDDON“ (Habersack et al.). Derzeit bestehen keine einheitlichen Untersuchungsansätze zum Sedimenttransport der Donau in Österreich und Ungarn. Deshalb werden in dem grenzüberschreitenden EU-EFRE-Kooperationsprojekt SEDDON (Sedimentforschung und Management an der Donau) Mess- und Modellierungssysteme für die Erfassung des Schwebstoffund Geschiebetransports verglichen und gemeinschaftliche Lösungsansätze erarbeitet. Weiters werden vorhandene Laboreinrichtungen für physikalische Modellversuche evaluiert und es wird in Wien ein Forschungsgerinne mit einem freien Durchfluss von bis zu $10 \mathrm{~m} 3 / \mathrm{s}$ ohne Pumpen gebaut, das gemeinsame Laborversuche zur Sedimentthematik ermöglicht.

Auch auf internationaler Ebene gewinnt die Fragestellung Feststoffhaushalt und Sedimenttransport an Bedeutung. Dies zeigt sich in diesem Heft im letzten Beitrag „SedAlp - Sediment management in Alpine basins: integrating sediment continuum, risk mitigation and hydropower" von Aigner et al. Das im EFRE-Alpine-Space-Programm laufende gleichnamige Projekt schließt die Alpenländer Frankreich, Italien, Slowenien, Österreich und Deutschland ein, die Schweiz ist als "observer" eingebunden. SedAlp trägt zu einem integrierten Management des Sedimenttransportes in alpinen Einzugsgebieten bei. Dabei geht es um eine effektive Reduktion von sedimentbezogenen Risiken, die Verbesserung der Fließgewässerökosysteme und die Reduktion der Auswirkungen der Wasserkraft auf den Feststoffhaushalt. Dabei werden Pilotaktivitäten in repräsentativen alpinen Einzugsgebieten der involvierten Länder umgesetzt, vergleichende Beiträge zum Sediment- und Schwemmholzmonitoring geleistet, Maßnahmen zur Verbesserung des Sedimentmanagements diskutiert und Empfehlungen zur Verbesserung der Sedimentkontinuität in Alpinen Flusseinzugsgebieten ausgearbeitet.

\section{Univ.-Prof. DI Dr. H. Habersack}

UNESCO-Lehrstuhl für Integrated River Research and Management,

Leiter Christian Doppler Labor für Innovative Methoden in Fließgewässermonitoring,

Modellierung und Flussbau, Institut für Wasserwirtschaft, Hydrologie und konstruktiven

Wasserbau, Department für Wasser - Atmosphäre - Umwelt,

Universität für Bodenkultur Wien,

Muthgasse 107,

1190 Wien, Österreich

E-Mail: helmut.habersack@boku.ac.at 